\title{
Intrusion of orthographic knowledge on phoneme awareness: Strong in normal readers, weak in dyslexic readers
}

\author{
KARIN LANDERL \\ University of Salzburg \\ UTA FRITH \\ MRC Cognitive Development Unit, London \\ HEINZ WIMMER \\ University of Salzburg
}

ADDRESS FOR CORRESPONDENCE

Karin Landerl, Department of Psychology, University of Salzburg, Hellbrunnerstr. 34, A-5020 Salzburg, Austria

\begin{abstract}
In three typical phonological awareness tasks it was found that children with normal reading development sometimes give responses that are based on orthographic rather than phonological information. In dyslexic children, the number of occurrences of such orthographic intrusions was significantly lower. This effect cannot be explained by positing a lower degree of orthographic knowledge in dyslexic children since a group of younger children who had the same spelling level as the dyslexics also showed more orthographic intrusions. A plausible explanation for this difference between normal and dyslexic readers is that, in normal readers, phonological and orthographic representations of words are so closely connected that they are usually coactivated, even if such a coactivation is misleading. In dyslexics this connection is less strong, so that orthographic representations interfere less with phonemic segmentation. The relevance of this finding with respect to recent assumptions about the importance of phonology in establishing orthographic representations is discussed.
\end{abstract}

Phonological awareness tasks have become a stock-in-trade in reading research, and yet these tasks are still poorly understood. Since Bruce (1964) introduced the phoneme deletion paradigm ("Can you say TENT without the tuh?"), innumerable studies have used this and similar tasks (e.g., "How many sounds are there in the word TEN?"). In general, these studies have confirmed Bruce's original finding, namely, that increased reading competence is reflected in improved performance on phoneme awareness tasks. Thus, 5-year-old English-speaking children do relatively poorly on pho-

() 1996 Cambridge University Press 0142-7164/96 $\$ 9.00+.10$ 
neme deletion tasks, but by age 9 they are at ceiling. Because the stimulus presentation in these tasks is auditory and in theory does not depend on reading ability, these tasks have been used with illiterates (Morais, Cary, Alegria, \& Bertelson, 1979), nonalphabetic readers (Read, Zhang, Nie, \& Ding, 1986), and prereaders. In these groups performance tends to be poor, and consequently debates have arisen about the mutual interaction of alphabetic knowledge and phoneme awareness (The Onset of Literacy, 1986; Segmental Analysis of Literacy, 1987).

The phenomenon that, in skilled adult readers, orthographic information intrudes and interferes with performance on auditory tasks such as rhyme judgment was first shown by Seidenberg and Tanenhaus (1979) and Donnenwerth-Nolan, Tanenhaus, and Seidenberg (1981). These important studies suggested that, in skilled adult readers, phonological and orthographic information in words are closely connected, so that these two types of representation are automatically coactivated, even when this is not advantageous, as in a rhyme judgment task. The question then arose as to whether such a close link between orthographic and phonological representations also exists for beginning readers.

Ehri and Wilce (1980) used a phoneme counting task with fourth graders where they compared stimulus words such as R-I-CH and P-I-TCH, which have the same number of phonemes but a different number of graphemes. They found that, just like the adults in Seidenberg and Tanenhaus's (1979) study, young readers' phonological judgment was based on orthographic representations. Thus, they heard more sounds in PITCH than in $\mathrm{RICH}$, even though phonetically they have the same number of sounds. Subsequently, similar findings were reported even for first graders (Barron, 1994; Tunmer $\&$ Nesdale, 1982). Thus, we can conclude that there is early orthographic interference in phonemic awareness tasks.

A recent study by Bruck (1992) examined the link between phonological and orthographic representations in dyslexics. Surprisingly, her findings suggest that, for dyslexics at all ages, this link is less strong than in normal readers. Bruck confronted normal and dyslexic readers with two conditions of a phoneme counting and a phoneme deletion task. One condition consisted of nonwords that contained the same number of letters as phonemes (e.g., TISK) and the other condition consisted of nonwords that contained one digraph so that these items contained more letters than phonemes (e.g., OTH contains three letters but only two phonemes). In accordance with other studies (Bradley \& Bryant, 1978; Bruck, 1992; Manis, Szeszulski, Holt, \& Graves, 1988; Olson, Wise, Connors, \& Rack, 1990), Bruck found that, on the nondigraph items, dyslexics made significantly more errors than reading level matched children. The interesting finding, with respect to the link between phonological and orthographic representations, was that normal readers were more misled by the way the digraph nonwords would be spelled than were the dyslexics. On the phoneme counting task, the large majority (between 83 and $99 \%$ ) of normal reader's errors were overshoots, that is, overestimations of the number of sounds in line with the number of letters. For the dyslexics, however, the percentage of over- 
shoots was considerably lower (between 51 and $77 \%$ ). Similarly, on the phoneme deletion task, the normal readers showed a stronger tendency than the dyslexics to delete only one letter of the digraph instead of the complete phoneme (e.g., THOACE becomes HOACE). On the basis of these data, Bruck (1992) concluded that dyslexics did not use orthographic information to the same extent as normal readers, suggesting that there is an independence between orthographic and phonological codes.

These findings are extremely interesting with respect to recent conceptions of the orthographic lexicon as a store of phonologically underpinned orthographic representations (Ehri, 1992; Perfetti, 1992; Stuart \& Coltheart, 1988). Phonological underpinning means that there are close connections between graphemes and grapheme strings and the according phonological segments, so that phonology is always coactivated if a word spelling is presented. The evidence on orthographic intrusions in phoneme awareness tasks shows that, in the normal reader, coactivation also takes place the other way around, that is, orthography is coactivated when phonological judgments must be made. Interestingly, for dyslexic readers there seems to be less coactivation, presumably in both directions.

However, other evidence somewhat contradicts Bruck's findings. Rack (1985), for example, suggests that dyslexics use orthographic information as a kind of compensatory strategy for their poor phonological processing and therefore rely more on orthographic information in their phonological judgment than do normal readers. In his study, dyslexic children needed more time to decide whether two auditorily presented words rhymed for word pairs that were orthographically dissimilar (FOOD-SHOULD) than for word pairs that were orthographically similar (GOOD-STOOD). Unexpectedly, the group of reading age control children in Rack's study did not show this effect. This is surprising in light of the findings of orthographic interference even in very young normal readers and leaves doubts about the validity of Rack's data.

Campbell and Butterworth (1985) found evidence for the use of an orthographic compensatory strategy in a single case study of a highly educated, adult dyslexic subject. While performance on a variety of phonemic awareness tasks was still poor, the strategy the subject used appeared to be based on orthographic information. However, the subject that Campbell and Butterworth examined was highly literate and may therefore not be typical of dyslexics in general.

Finally, Perin (1983) argued that she found evidence for the influence of orthographic knowledge on phonological awareness tasks for both normal readers and children with reading problems. Three groups of children (i.e., good readers/good spellers, good readers/poor spellers, and poor readers/ poor spellers) were asked to count the number of phonemes in mismatching words (which had more letters than phonemes), matching words (which had a one-to-one phoneme-letter match), and nonwords derived from the latter. Perin analyzed the children's performance on those items for which it was clear that they had an orthographic representation (i.e., they could produce a correct spelling) and found no reliable interaction between word 
type and group. All three groups may have used orthographic knowledge in order to solve the task. However, an inspection of the error percentages shows some difference between normal children's and reading- and spellingdisabled children's performances. While, for the good readers/good spellers, the error percentage for the mismatching words was considerably higher than for the other two word types $(37 \%$ vs. $22 \%)$, there was less difference between the mismatching words and the other two word types for the poor readers/poor spellers $(41 \%$ vs. $37 \%$ ). In addition, Perin found that, on a spoonerism task, the normal readers showed a stronger influence of orthographic knowledge than the children with reading and/or spelling problems. Normal readers gave more incorrect responses than the other two groups on those items where the initial phonemes were mapped by letter clusters rather than single letters (e.g., CHUCK BERRY became /bnk keri:/ instead of /bsk t $\mathrm{feri} / /)$. Thus, these data confirm Bruck's assumption that there is less coactivation of the orthographic code in phoneme awareness tasks in dyslexic than in normal readers.

In view of its relevance for theories of dyslexia, the present study attempts to explore further the extent to which orthographic information intrudes on dyslexic children's performance on phoneme awareness tasks. We made several methodological modifications to Bruck's (1992) study. First, a well-defined sample of dyslexic children was examined using a main comparison group of spelling level matched children. Spelling level is probably a better indicator of children's knowledge of orthographic patterns than reading level, since to be able to provide a correct spelling of a particular word in English, the exact letter-by-letter knowledge of the word is necessary. In addition, we required that the two groups of dyslexic and spelling level matched children were not only comparable on a standardized spelling test, but also able to produce the same number of correct spellings for the words that we used as stimuli in the phonological awareness tasks. Third, we used words rather than nonwords. Words of high frequency were used so that there was a good chance that the subjects would know the word spellings (i.e., have accurate orthographic representations). Further, we compared sets of rhyming stimuli that were similar phonologically but different orthographically (LORD vs. SWORD). And finally, whereas Bruck (1992) examined normal and dyslexic subjects' performance on items including a digraph, we expected our subjects to be misled by silent letters. A series of studies by Ehri and Wilce (1982) showed that silent letters are salient in memory representations of word spellings. Thus, we reasoned that overshoot errors might be readily provoked for words including a silent letter. Since our hypothesis predicts a lack of overshoot errors in dyslexics, it was important to create a strong temptation for such errors. However, we very carefully explained to the children, through the practice examples, that the tasks were about sounds and not about letters.

Three typical, widely used phonological awareness tasks - a phoneme counting task and two phoneme deletion tasks - were administered. Each task consisted of a control condition, which included words that were phonologically transparent (e.g., HAM, HOT) but could contain regular di- 
Landerl et al.: Orthographic intrusions

Table 1. Mean scores of subject characteristics, with ranges in parentheses

\begin{tabular}{lccl}
\hline & \multicolumn{1}{c}{ Dyslexics } & SA-controls & \multicolumn{1}{c}{ CA-controls } \\
\hline$N$ & 14 & 11 & 13 \\
Age & $12 ; 3(10 ; 8-13 ; 8)$ & $8 ; 2(7 ; 6-9 ; 1)$ & $13 ; 1(12 ; 6-13 ; 6)$ \\
Reading age & $8 ; 7(7 ; 2-10 ; 4)$ & $8 ; 4(7 ; 4-9 ; 4)$ & $14 ; 1(12 ; 5-14 ; 5)$ \\
Spelling age & $8 ; 1(7 ; 3-9 ; 3)$ & $8 ; 6(7 ; 5-9 ; 5)$ & $13 ; 8(12 ; 5-14 ; 5)$ \\
Raven IQ (percentile) & $67(30-92)$ & $68(40-97)$ & $93(77-93)$ \\
\hline
\end{tabular}

graphs (e.g., ROOF, BATH), ' and a silent letter condition, which included words that had a phonologically obsolete silent letter (e.g., LAM If task performance is influenced by the knowledge of orthographic representations, then the silent letter should emerge in the children's responses. If, however, dyslexics are not distracted by their orthographic knowledge to the same extent as normal readers, then they should show a smaller number of these orthographic intrusions.

\section{METHOD}

\section{Subjects}

All together, 38 children participated in this study. The characteristics of the subjects are presented in Table 1. A group of dyslexic children consisted of 14 boys with a mean age of 12;3. Reading and spelling level were assessed by the British Ability Scales, Word Reading scale (Elliot, Murray, \& Pearson, 1983) and the British Ability Scales Spelling scale (Elliot, 1992), respectively. The dyslexics achieved a mean reading age of $8 ; 7$ and a mean spelling age that was somewhat lower (i.e., 8;1). A group of chronological age matched children (CA-controls) consisted of 13 boys with a mean age of $13 ; 1$, a reading age of $14 ; 1$, and a spelling age of $13 ; 8$. Another group of control children was matched by spelling age (SA-controls). This group consisted of 11 children ( 9 boys, 2 girls) with a mean spelling age of $8 ; 6$ and a mean reading age of $8 ; 4$. These children were considerably younger than the dyslexics $(M=8 ; 2)$. The children's IQ was assessed by the Raven's Standard Progressive Matrices (Raven, 1987). From Table 1 it is obvious that the mean IQ percentiles of all three groups were above average; however, coincidentally, the CA-controls' IQ percentile scores turned out to be considerably higher than those of the dyslexics and the SA-controls. This difference in nonverbal intelligence creates an even stronger test for the influence of orthographic information on phonological awareness tasks because it rules out the possibility that the CA-control children, who, according to Bruck (1992), are prone to commit orthographic intrusion errors, may misunderstand the explicitly given instruction to count or delete sounds and not letters. 
Landerl et al.: Orthographic intrusions

Phonological tasks

Three typical phonological awareness tasks were used (i.e., phoneme counting, deletion of the first phoneme, and deletion of the last phoneme). In order to examine the influence of the knowledge of orthographic representations on the performance on these tasks, two conditions were constructed for each task. In the control condition, words with phonologically transparent spellings were presented (e.g., HAM, HOT). The words in the silent letter condition were phonologically similar to (i.e., rhymed with) the control items; however, they included a letter that is phonologically obsolete (e.g., LAMB, WHAT). If children use their phonological representations to perform phonological awareness tasks, then these silent letters should have no influence at all. If, however, children use their knowledge of orthographic representations, then a typical kind of error should occur, which we called "orthographic intrusions." In the phoneme counting task, the typical orthographic intrusion would be that a child counts the silent letter as a phoneme and therefore overestimates the number of sounds in the presented word (e.g., "LAMB: /1/-/æ/-/m/-/b/ - four sounds"; "HALF: /h/-/a/-/1/-/f/ four sounds"). For the two deletion tasks, the words for the silent letter condition were chosen in such a way that the silent letter was in the relevant position, that is, in second position for deletion of the first phoneme and in penultimate position for deletion of the last phoneme. An orthographic intrusion would be present if the phoneme corresponding to the silent letter emerges in the child's pronunciation of the target word without the first, respectively, final phoneme: for example, /hot/ instead of /ot/ for WHAT or /wo:d/ instead of /o:d/ for swORD for deletion of the first phoneme; $/ \mathrm{kal} /$ instead of $/ \mathrm{ka} /$ for CALF or $/ \mathrm{kæt} /$ instead of $/ \mathrm{kæ} /$ for CATCH for deletion of the last phoneme.

Each condition consisted of 7 one-syllable words. All of the words could easily be illustrated by a picture and were expected to be familiar to children of about 7 years of age. The words for the control condition were chosen so that each one was phonologically similar to a word in the silent letter condition, namely, the syllable rimes of the two words were pronounced identically (e.g., LAMB - HAM) or differed in only one phoneme (e.g., CALF - BATH). Each task was introduced by five practice examples.

The practice examples were also used to demonstrate that sounds and letters are not equivalent. For example, the first practice example for phoneme counting was MISS. The experimenter explained that MIsS consists of four letters but only of three sounds, $/ \mathrm{m} /, / \mathrm{i} /$, and $/ \mathrm{s} /$. It is important to note that children were told explicitly that the tasks had to do with sounds in words, not with letters. For further clarification of what was meant by "sound," the practice examples included words with consonant clusters. The children who interpreted the consonant clusters as one sound were corrected and told that, for example, wIND without the last sound is win, not wi. Feedback was given for the practice trials, but not for the experimental trials. However, some filler words, for which feedback was given, were presented in between the experimental items. These filler words seemed to 
be necessary because the pilot work showed that some children, despite practice, tended to adopt strategies such as "delete everything before the vowel" or "delete everything after the vowel" for the two deletion tasks. The fillers were words for which such strategies led to incorrect responses (e.g., GLASS for deletion of the first sound); children who gave incorrect responses for these fillers were corrected and instructed again. Practice items, experimental items, and filler words for all three tasks are listed in the Appendix.

For the two deletion tasks, the children had to answer the standard question, "What do you get if you drop the first/last sound in . . ?" For phoneme counting, they were asked to place one coin on the table for each sound. At the same time, the child had to name the sounds. An answer was only scored as correct if the child put the correct number of coins on the table and named the correct sounds. Asking the child to name the sounds in addition to putting down coins had a major advantage in that the experimenter could observe if the task was performed in a correct manner or if an orthographic intrusion occurred. Two random sequences of items were constructed for each task, with the restriction that the phonologically similar words of the control and the silent letter condition should not be presented in immediate succession. Each of the sequences was presented to half of the subjects. The three tasks were presented in two different sequences; half of the children were given phoneme counting before deletion of the first sound, followed by deletion of the last sound; the other half got phoneme counting after deletion of the first sound and deletion of the last sound. For each item, a picture was presented to clarify the meaning of the word.

\section{Word spelling}

In order to check the children's knowledge of the orthography of the experimental items, they were asked to spell the words after the three phonological awareness tasks were performed.

\section{RESULTS}

In a first step, the total number of errors in the control and the silent letter conditions were analyzed. Since the number of errors tended to be rather low on each of the three tasks (the maximum was 2.5 out of 7 in phoneme counting for CA- and SA-controls), the scores were combined. This seems to be justified by the result of an ANOVA, which showed that neither group nor condition interacted with task, $F(4,70)=4.83$, and $F(2,70)=$ 0.87 , for the interaction terms $(p>.10)$. However, the correlations between the scores of the three tasks were rather low (between .17 and .34), presumably because of the ceiling effect.

It should be noted that this total error score includes segmentation errors as well as orthographic intrusions. From the phonemic segmentation component, it follows that the dyslexic children would be expected to commit 
Landerl et al.: Orthographic intrusions

Table 2. Mean number (SD) of errors in the control and silent letter conditions (max. = 7)

\begin{tabular}{lccc}
\hline \hline & Dyslexics & SA-controls & CA-controls \\
\hline Control condition & $1.9(1.4)$ & $4.1(3.0)$ & $0.4(0.7)$ \\
Silent letter condition & $3.4(2.8)$ & $5.9(4.4)$ & $4.8(2.2)$ \\
\hline
\end{tabular}

more errors than the control children in the control condition, which, presumably, should not give rise to orthographic intrusion errors. The silent letter condition should lead to additional errors caused by orthographic intrusions. The expectation is that, for the control children, there will be a stronger tendency to commit orthographic intrusion errors than for the dyslexics. Therefore, in the silent letter condition, the difference in total number of errors (segmentation errors plus orthographic intrusions) between the dyslexic and control children should be diminished when compared with the difference in the control condition. In the extreme case, one might expect that, in the silent letter condition, the control children might actually exhibit a higher number of total errors than the dyslexic children. Table 2 contrasts the total number of errors for the control condition and the silent letter condition for each of the three groups.

From Table 2, it is evident that the formulated predictions were valid for the comparison of dyslexic children and CA-controls. In a 2 (Condition) $\times$ 2 (Group) ANOVA comparing dyslexics and CA-controls, the interaction between group and condition proved to be reliable, $F(1,25)=13.9, p<$ .001 . As expected, in the control condition (provoking phoneme segmentation errors), the dyslexic children committed more errors than the control children. This difference was reliable: planned contrast, $t(25)=2.7, p<$ .05 . The expectation that dyslexic children would be less induced to commit orthographic intrusions was confirmed by the finding that, in the silent letter condition (provoking orthographic intrusions in addition to phoneme segmentation errors), the dyslexics committed even fewer errors than the CA-controls: planned contrast, $t(25)=2.5, p<.05$. The main effect of condition was also significant, $F(1,25)=56.1, p<.001$, but there was no reliable difference between the two groups, $F(1,25)<1$.

For the comparison of dyslexics with SA-controls, the means in Table 2 present a completely different pattern. The fact that SA-controls showed a higher number of errors than the dyslexics in the simple condition, $t(23)=$ $2.4, p<.05$, already contradicts the well-established finding that dyslexic children have more problems with phonemic segmentation than normal readers of the same reading level. However, a closer inspection of SAchildren's errors shows that the control condition provoked orthographic intrusions for them as well. It should be noted that the control items included a number of words with digraphs, and it was these that provoked typical orthographic errors. Examples of such errors were: /lin/ instead of /lai/ when asked to delete the last phoneme of LINE, /bæt/ instead of 
Landerl et al.: Orthographic intrusions

Table 3. Percentage of orthographic intrusions for matching, digraph, and silent letter items

\begin{tabular}{llrr}
\hline \hline & Dyslexics & SA-controls & CA-controls \\
\hline Control condition & & & \\
Matching items & $0,0(0,0)$ & $3,6(5,1)$ & $0,0(0,0)$ \\
Digraph items & $3,3(7,7)$ & $19,8(19,4)$ & $3,5(5,9)$ \\
Silent letter condition & $6,5(9,8)$ & $26,0(20,2)$ & $22,0(9,8)$ \\
\hline \hline
\end{tabular}

/ba/ when asked to delete the last phoneme of BATH, or counting four sounds $(/ \mathrm{r} /-/ \mathrm{i} /-/ \mathrm{k} /-/ \mathrm{h} /)$ in $\mathrm{RICH}$. To overcome this obvious problem in item selection, the items of the control condition were recategorized in matching items which had a one-to-one correspondence between phonemes and letters and digraph items (marked with an asterisk in the Appendix).

Our general expectation was that dyslexic children should show a lower number of orthographic intrusions than the control children. To create a more straightforward test of this hypothesis, the following analysis is based on the number of orthographic intrusions only and not on total number of errors, which also includes phoneme segmentation errors. Table 3 presents the percentage of orthographic responses in each of the three conditions. Only those responses that were clearly based on the spelling were counted as orthographic intrusions. Responses such as $/ \mathrm{t} /-/ \mathrm{i} /-/ \mathrm{i} /$ when asked to count the phonemes in the word TEA were considered ambiguous and were not counted as orthographic intrusions. This response, for instance, might be based on the orthographic representation of the word as well as on an incorrect segmentation of the phoneme sequence /ti:/.

In a Group (3) $\times$ Type (matching vs. digraph vs. silent letter) ANOVA with repeated measures, both main effects and the interaction proved to be reliable: group, $F(2,35)=8.0, p<.001$; type, $F(2,70)=41.4, p<$ .001 ; Group $\times$ Type, $F(4,70)=6.6, p<.001$. Table 3 shows that hardly any orthographic intrusions became evident in the matching condition. Two SA-control children counted correctly the number of sounds in HAM, but obviously named the letters instead of the sounds ("HAM - / eit J/-/ei//em/"). Another two SA-control children responded "/go/" when asked to delete the last sound in GOOD. In the digraph condition, it was mainly the SA-controls who were misled by orthography. Planned contrasts showed that the SA-controls committed significantly more orthographic intrusions than the dyslexic children, $t(70)=5.1, p<.05$. In the silent letter condition, both of the control groups showed significantly more orthographic intrusions than the dyslexic children: CA-controls, $t(70)=5.0$; SAcontrols, $t(70)=6.0, p<.05$.

The difference in orthographic intrusion errors between dyslexics and SA-control children cannot be attributed to a difference in their knowledge of spelling because their performances on the word spelling task were very similar. In the silent letter condition, the dyslexic children produced, on 
average, 9.0 correct spellings, compared with 8.6 correct spellings for the SA-control children. For the control condition, the numbers were 16.7 and 17.0 , respectively.

The higher number of orthographic intrusions in CA-controls compared with dyslexic children could be due to their superior spelling knowledge, since their performance on the spelling task was at ceiling. However, even when the number of correct spellings was introduced as a covariate, the interaction between condition and group was still significant, $F(2,50)=$ $14.3, p<.001$.

In a final step, we analyzed those erroneous responses which differed from the correct phonological analysis, but apparently were not caused by a knowledge of the spelling. Examples of such errors were: /oz/ when asked to delete the first sound of $200, / \mathrm{br} /$ when asked to delete the last sound of BRIDGE, or putting two coins on the table and saying, /ha/-/f/ when asked to count the sounds in the word HALF. Combined over all conditions, the dyslexic children committed a higher number of these errors $(M=3.6)$ than both the CA-controls $(M=0.6), t(25)=4.1, p<.001$, and the SA-controls $(M=1.6), t(23)=2.1, p<.05$.

\section{DISCUSSION}

The results of this study confirm the interesting finding by Bruck (1992): in phonological awareness tasks, normal readers are heavily distracted by the knowledge of word spellings, while, for dyslexics, the distraction is less strong. In three typical, widely used phonological awareness tasks, dyslexics showed a significantly lower number of orthographic intrusions than either CA- or SA-control children. In contrast to Bruck, who looked at orthographic intrusions in the case of digraph items, we used words that included silent letters. Previous research (Ehri \& Wilce, 1982) showed that silent letters have a prominent position in orthographic representations of words. Thus, we assumed that silent letters should be more misleading than digraphs, which are often taught as single units in grapheme-to-phoneme correspondences (e.g., $e e=/ \mathrm{i}: /$ ) and are therefore more likely to be stored as such. Our assumption turned out to be correct for the 12-year-old CAcontrol children, who showed almost no orthographic intrusions for the digraph items. The younger SA-controls, however, were misled by digraphs to an equal extent as by silent letters. It seems that, for young readers, word spellings are such a prominent representation of words that sometimes they cannot avoid thinking about the letters, even though they are explicitly instructed to reflect on the sound structure of a word. With increasing experience, the child learns to represent digraphs as single units, so that by age 12 normal readers are no longer misled in their phonological judgments by digraphs; however, as our results show, they are still misled by silent letters.

The main finding was that the dyslexic children showed a significantly lower percentage of orthographic intrusions than either the CA- or SAcontrol groups. Both digraphs and silent letters turned out to be less 
misleading for dyslexic children. On the other hand, dyslexic children committed more of the other errors, pointing to difficulties in phoneme segmentation, than either control group. This is in agreement with other findings that have demonstrated that dyslexic children show impairments in phoneme awareness, not only in comparison with normal readers of the same age (Liberman, Shankweiler, Liberman, Fowler, \& Fischer, 1977; Wimmer, 1993), but also in some studies comparing dyslexics with younger, reading level matched children (Bradley \& Bryant, 1978; Bruck, 1992; Manis et al., 1988; Olson et al., 1990). However, the dyslexics in our study gave correct responses for $87 \%$ of all items. This may seem surprising in relation to Bruck's (1992) finding that adults with a childhood diagnosis of dyslexia continued to have difficulties with phonological awareness tasks. Of course, the tasks that we used cannot be compared with more difficult phoneme awareness tasks such as pig latin (Pennington, Van Orden, Smith, Green, \& Haith, 1990) or spoonerisms (Campbell \& Butterworth, 1985; Perin, 1983). Our phoneme counting task consisted of CVC-items and excluded consonant clusters, which are usually somewhat more difficult to segment. For the two deletion tasks, it was also the case that the child never had to delete a consonant from a consonant cluster. Only single consonants followed or preceded by a vowel had to be deleted. The level of task difficulty would be expected to have a strong influence on the dyslexics' performance.

As we pointed out, there is independent evidence that normal children's and adults' phonological judgments are influenced by their orthographic knowledge (Donnenwerth-Nolan et al., 1981; Ehri, 1984; Ehri \& Wilce, 1980; Seidenberg \& Tanenhaus, 1979; Tunmer \& Nesdale, 1982). A major criticism of many of these studies is that the subjects may have misinterpreted the phonological awareness tasks as letter counting tasks. This criticism, however, is not valid for the present study. We strongly discouraged our subjects from using letters and explained in great detail the difference between letters and sounds. The strikingly high intelligence of the CAcontrol children makes it even less likely that they failed to understand our instruction. Nevertheless, we again confirmed the influence of orthographic information on phonological judgments for both of our control samples (the 8-year-olds as well as the 12-year-olds); however, we did not find this effect to the same extent in our dyslexic sample. The lesser degree of distraction by orthography in dyslexics is not just a consequence of poor reading and spelling experience. This was demonstrated by the fact that the SAcontrols also showed more orthographic intrusions than the dyslexics.

This finding is interesting with regard to recent theories about the structure and development of the orthographic lexicon (Ehri, 1992; Perfetti, 1992; Rack, Hulme, Snowling, \& Wightman, 1994; Stuart \& Coltheart, 1988). These theories suggest that the orthographic lexicon is phonologically underpinned (Perfetti, 1992), and that the connections between word spellings and phonological representations are necessary to set up an efficient and automatically accessible orthographic lexicon (Ehri, 1992; Rack et al., 1994). The study by Bruck (1992), as well as our own have shown 
Landerl et al.: Orthographic intrusions

that the normally found interference between orthographic and phonological representations is less in evidence for dyslexic than for normal readers. We suggest that, in the dyslexics' mental lexicon, there is less connection between orthographic and phonological representations. We have reason to believe that dyslexics acquire word spellings in a way that is different from normal readers, namely, with less phonological underpinning.

In conclusion, we suggest that a weak link between phonological and orthographic representations might be a central problem in dyslexia. Thus, seeing a written word does not automatically evoke the word's inner sound. Moreover, the sound of a word does not automatically evoke the inner orthographic image. It may be that it is this automatic coactivation that makes phoneme awareness tasks so easy for alphabetically literate people even though the same mechanism makes them susceptible to unwanted interference. Ironically, under certain conditions, the hypothesized disconnection enables dyslexics to outperform normal readers on phonological awareness tasks.

\section{APPENDIX}

Stimuli of the Phonological Awareness Tasks

\begin{tabular}{|c|c|c|c|}
\hline & \multirow[b]{2}{*}{ Phoneme counting } & \multicolumn{2}{|c|}{ Phoneme deletion } \\
\hline & & First phoneme & Last phoneme \\
\hline \multirow[t]{5}{*}{ Practice items } & MISS & SHIP & MESS \\
\hline & FOOT & SPOT & WIND \\
\hline & BLUE & FRAME & BEAST \\
\hline & SKIN & PULL & STOP \\
\hline & MOON & BRUSH & HOLD \\
\hline \multirow[t]{3}{*}{ Filler items } & GOLF & GLASS & MILK \\
\hline & LAMP & BLACK & LIST \\
\hline & COLD & SWIM & TENT \\
\hline \multirow{7}{*}{ Control condition } & ROOF* & MOST & BATH* \\
\hline & HAM & TAIL & $\mathrm{MUCH}^{*}$ \\
\hline & WOOD* & LORD & LINE* \\
\hline & TEA * & TINY & GOOD \\
\hline & LESS* & HOT & WHITE* \\
\hline & PAGE* & $\mathrm{ZOO}$ & SHOUT \\
\hline & RICH* & HEN & STAGE* \\
\hline \multirow[t]{7}{*}{ Silent letter condition } & HALF & GHOST & CALF \\
\hline & LAMB & WHALE & CATCH \\
\hline & WOULD & SWORD & SIGN \\
\hline & KNEE & RHINO & COULD \\
\hline & GUESS & WHAT & LIGHT \\
\hline & HEDGE & TWO & DOUBT \\
\hline & WITCH & WHEN & BRIDGE \\
\hline
\end{tabular}

*Digraph items in the control condition. 
Landerl et al.: Orthographic intrusions

\section{NOTE}

1. The present study was actually designed independently from Bruck's research. The testing was carried out before Bruck's data were published. This explains why we were not concerned about including digraphs in our control condition. In the statistical analysis, the control items will be recategorized into matching items with a one-to-one correspondence between letters and phonemes and digraph items.

\section{REFERENCES}

Barron, R. W. (1994, October). Proto-literate knowledge: Antecedents and influences on phonological awareness and literacy. Paper presented at the North Atlantic Treaty Organization Advanced Study Institute: Cognitive and linguistic bases of reading, writing, and spelling. Alvor, Portugal.

Bradley, L., \& Bryant, P. E. (1978). Difficulties in auditory organization as a possible cause of reading backwardness. Nature, 27l, 746-747.

Bruce, D. J. (1964). The analysis of word sounds. British Journal of Educational Psychology, 34, 158-170.

Bruck, M. (1992). Persistence of dyslexics' phonological awareness deficits. Developmental Psychology, 28, 874-886,

Campbell, R., \& Butterworth, B. (1985). Phonological dyslexia and dysgraphia in a highly literate subject: A developmental case with associated deficits of phonemic processing and awareness. Quarterly Journal of Experimental Psychology, 37, 435-475.

Donnenwerth-Nolan, S., Tanenhaus, M. K., \& Seidenberg, M. S. (1981). Multiple codeactivation in word recognition: Evidence from rhyme monitoring. Journal of Experimental Psychology: Human Learning and Memory, 7, 170-180.

Ehri, L. C. (1984). How orthography alters spoken language competencies in children learning to read and spell. In $\mathrm{J}$. Downing \& $\mathrm{R}$. Valtin (Eds.), Language awareness and learning to read (pp. 119-147). New York: Springer-Verlag.

(1992). Reconceptualizing the development of sight word reading and its relationship to recoding. In P. B. Gough, L. C. Ehri, \& R. Treiman (Eds.), Reading acquisition (pp. 107-144). Hillsdale, NJ: Erlbaum.

Ehri, L. C., \& Wilce, L. S. (1980). The influence of orthography on readers' reconceptualisation of the phonemic structure of words. Applied Psycholinguistics, 1, 371-385.

(1982). The salience of silent letters in children's memory for word spellings. Memory and Cognition, 104, 155-166.

Elliot, C. D. (1992). British Ability Scales: Spelling Scale. Windsor, Berkshire: NFERNELSON.

Elliot, C. D., Murray, D. J., \& Pearson, L. S. (1983). British Ability Scales: Word Reading. Windsor, Berkshire: NFER-NELSON.

Liberman, I. Y., Shankweiler, D., Liberman, A. M., Fowler, C., \& Fischer, F. W. (1977). Phonetic segmentation and recoding in the beginning reader. In A. S. Reber \& D. L. Scarborough (Eds.), Towards a psychology of reading (pp. 207-255). Hillsdale, NJ: Erlbaum.

Manis, F. R., Szeszulski, P. A., Holt, L. K., \& Graves, K. (1988). A developmental perspective on dyslexia subtypes. Annals of Dyslexia, 38, 139-153.

Morais, J., Cary, L., Alegria, J., \& Bertelson, P. (1979). Does awareness of speech as a sequence of phones arise spontaneously? Cognition, 7, 323-331.

Olson, R., Wise, B., Connors, F., \& Rack, J. (1990). Organisation, heritability, and remediation of component word recognition and language skills in disabled readers. In T. Carr \& B. A. Levy (Eds.), Reading and its development: Component skills approaches (pp. 261-322). London: Academic.

Pennington, B. F., Van Orden, G. C., Smith, S. D., Green, P. A., \& Haith, M. M. (1990). Phonological processing skills and deficits in adult dysiexics. Child Development, 61, $1753-1778$.

Perfetti, C. A. (1992). The representation problem in reading acquisition. In P. B. Gough, 
Landerl et al.: Orthographic intrusions

L. C. Ehri, \& R. Treiman (Eds.), Reading aquisition (pp. 145-174). Hillsdale, NJ: Erlbaum.

Perin, D. (1983). Phonemic segmentation and spelling. British Journal of Psychology, 74, 129-144.

Rack, J., Hulme, C., Snowling, M., \& Wightman, J. (1994). The role of phonology in young children learning to read words: The direct mapping hypothesis. Journal of Experimental Child Psychology, 57, 42-71.

Rack, J. P. (1985). Orthographic and phonetic coding in developmental dyslexia. British Journal of Psychology, 76, 325-340.

Raven, J. C. (1987). Manual for Raven's Progressive Matrices and Vocabulary Scales, Section 3. Standard Progressive Matrices. London: H. K. Lewis \& Co.

Read, C., Zhang, Y., Nie, H., \& Ding, B. (1986). The ability to manipulate speech sounds depends on knowing alphabetic writing. Cognition, 24, 31-34.

Segmental Analysis and Literacy [special issue]. (1987). European Bulletin of Cognitive Psychology, 7(5).

Seidenberg, M. S., \& Tanenhaus, M. K. (1979). Orthographic effects on rhyme monitoring. Journal of Experimental Psychology: Human Learning and Memory, 5, 546-554.

Stuart, M., \& Coltheart, M. (1988). Does reading develop in a sequence of stages? Cognition, $30,139-181$.

The Onset of Literacy [special issue]. (1986). Cognition, 24(I/2).

Tunmer, W. E., \& Nesdale, A. R. (1982). The effects of digraphs and pseudowords on phonemic segmentation in young children. Applied Psycholinguistics, 3, 299-311.

Wimmer, H. (1993). Characteristics of developmental dyslexia in a regular writing system. Applied Psycholinguistics, 14, 1-33. 